\title{
A Novel Earplug System for Filtering Out Dental Noise
}

\section{Jiang-Ren Chang}

Associate Professor, Department of System Engineering and Naval Architecture, National Taiwan Ocean University, 2, Pei-Ning Road, Keelung, Taiwan 202, R.O.C., cjr@sena.ntou.edu.tw

Huan-Kun Cheng

Graduate Student, Graduate Institute of Oral Rehabilitation Sciences, Taipei Medical University

Sheng-Yang Lee

Professor, Dental Department of Wan-Fang Hospital; and Graduate Insitute of Oral Rehabilitation Sciences, Taipei Medical University

Mingsian R. Bai

Professor, Department of Mechanical Engineering, National Chiao Tung University

Rong-I Lean

Graduate Student, Graduate Institute of Oral Rehabilitation Sciences, Taipei Medical University

Follow this and additional works at: https://jmstt.ntou.edu.tw/journal

Part of the Engineering Commons

\section{Recommended Citation}

Chang, Jiang-Ren; Cheng, Huan-Kun; Lee, Sheng-Yang; Bai, Mingsian R.; and Lean, Rong-I (2003) "A Novel Earplug System for Filtering Out Dental Noise," Journal of Marine Science and Technology. Vol. 11: Iss. 4, Article 1.

DOI: $10.51400 / 2709-6998.2288$

Available at: https://jmstt.ntou.edu.tw/journal/vol11/iss4/1

This Research Article is brought to you for free and open access by Journal of Marine Science and Technology. It has been accepted for inclusion in Journal of Marine Science and Technology by an authorized editor of Journal of Marine Science and Technology. 


\title{
A NOVEL EARPLUG SYSTEM FOR FILTERING OUT DENTAL NOISE
}

\author{
Jiang-Ren Chang* Huan-Kun Cheng** Sheng-Yang Lee***, \\ Mingsian R. Bai**** and Rong-I Lean**
}

\begin{abstract}
Undergoing dental treatment is usually a very unpleasant experience for people who have toothaches, especially when they are hurting and there exists some intolerable mechanical noise. To reduce the noise and promote the work efficiency of dentists, a new noisefiltering earplug system with optional music was developed to filter out dental machine noise; if necessary, it can also be adopted as a solution to reduce pain and anxiety of patients during treatments. Furthermore, it can also simultaneously serve as a communication tool between the patient and dentist. In this study, various kinds of noises were initially collected through an off-line receiver and then connected to a personal computer. The software, CoolEdit and LABVIEW, were used to analyze the frequency ranges of the mechanical noises, and a cutoff frequency was identified through several comparisons. After the cutoff frequency was determined, the active noise-filtering system was designed with several circuits to filter out those frequencies higher than the cutoff frequency but reserving only the range of speech. In addition, music signals from an MP3 or CD player can also be transmitted into the earplug system for increasing the signal to noise ratio. The anxiety and tension of patients during treatment can thus be partially relieved by the euphonious music, and the pain of toothaches of patients might be further reduced. Moreover, when the voice signal from the dentist is transmitted into the patient's earplug, the musical signal will automatically reduced by $12 \mathrm{~dB}$ for convenient communication. It was found that the developed noisefiltering earplug system performed well in several tests, such as detecting various powers of signals by monitoring the waveforms, identifying variations in noise and speech signals before and after the use of the filter, and checking working conditions of the designed circuits.
\end{abstract}

Paper Submitted 09/12/03, Accepted 10/30/03. Author for Correspondence: Jiang-Ren Chang.E-mail: cjr@sena.ntou.edu.tw

*Associate Professor, Department of System Engineering and Naval Architecture, National Taiwan Ocean University, 2, Pei-Ning Road, Keelung, Taiwan 202, R.O.C.

** Graduate Student, Graduate Institute of Oral Rehabilitation Sciences, Taipei Medical University

***Professor, Dental Department of Wan-Fang Hospital; and Graduate Insitute of Oral Rehabilitation Sciences, Taipei Medical University

****Professor, Department of Mechanical Engineering, National Chiao Tung University
Sound is a ubiquitous component of our environment from which there is no escape and is also one of the principal media of communication between human beings. However, the adverse effects of excessive sound in causing hearing damage, raising stress levels, disturbing rest and sleep, reducing the efficiency of task performance, and interfering with verbal and musical communication, are widely experienced. Thus, noise pollution, defined as impurities of unpleasant sounds, has become vitally important to human beings, and much research has been carried out on determining ways to prevent or resolve such annoying noises [14].

For noise problems in the community, several investigations $[2,4,7]$ were conducted, and the problems were further comprehensively studied [25]. According to the work of Belojevic et al. [2] with an interview method concerning specific questions, the widely accepted scientific fact that living in a "black acoustic zone" $\left(\mathbf{L}_{\mathbf{e q}}>65 \mathrm{~dB}(\mathrm{~A})\right)$ places an urban population in a high-risk category for numerous subjective effects of noise, including psychological, sleep, and behavioral disorders, was further confirmed. Especially for the working environment of a hospital, those harmful effects not only interfere with medical treatments but have also influenced mental performance and occupational health hazards of those people exposed to a noisy environment [17-18, 26-27]. A recent experimental study [3] for assessing the mental performance of people with personality traits of intro-extroversion showed that extroverts performed faster, while introverts had more-pronounced subjective effects of annoyance, poor concentration, and fatigue during mental performances in noisy compared to quiet conditions. However, literature investigating the long-term effects of noise exposure on physiological and psychological behaviors of people is not available.

Nonetheless, it is well known that noise problems are inherent in every major hospital worldwide, causing 
increased amounts of anxiety, sleep loss, pain perception, and prolonged convalescence in every segment of the population [6]. According to Clark and Bohne [11], hearing loss suffered by approximately one-fourth of all Americans 65 and older is not part of the aging process, but largely due to excess noise on the auditory system. The damage, regardless of whether it is from short- and long-term exposure to noise, is evident by the loss of sensory cells on the organ of Corti [11]. In addition, McLean and Tarnopolsky [23] investigated the effects of noise on both humans and animals from the perspective of mental health and further proposed a relationship between increased systolic and diastolic blood pressure in patients with essential hypertension. Arvidsson and Lindvall [1] evaluated the relationships among noise, annoyance, mental performance ability, as well as hormone level, and their conclusions supported the hypothesis that the tendency to be annoyed by noise is associated with impaired performance in a generally stressful situation.

Noises arising from those rotating instruments used for endo-dentic treatments usually make patients anxious and may even pose serious occupational health hazards to technicians and dentists. At about $6 \%$ of 731 Swedish dental laboratory technicians complained of hearing problems due to tinnitus or phantom highfrequency sounds [19], and $3.4 \%$ of 220 Thai dentists reported that they had hearing problems [10]. Therefore, reducing unavoidable noise interference between dentists and patients was the initial motivation of the present study.

Apart from those noise problems during dental treatments, patients may present to dentists and complain of some physical symptoms such as a toothache, headache, and facial pain; only after much inappropriate treatment are these symptoms revealed to be due to emotional disturbances [15]. Thus, emotional strain
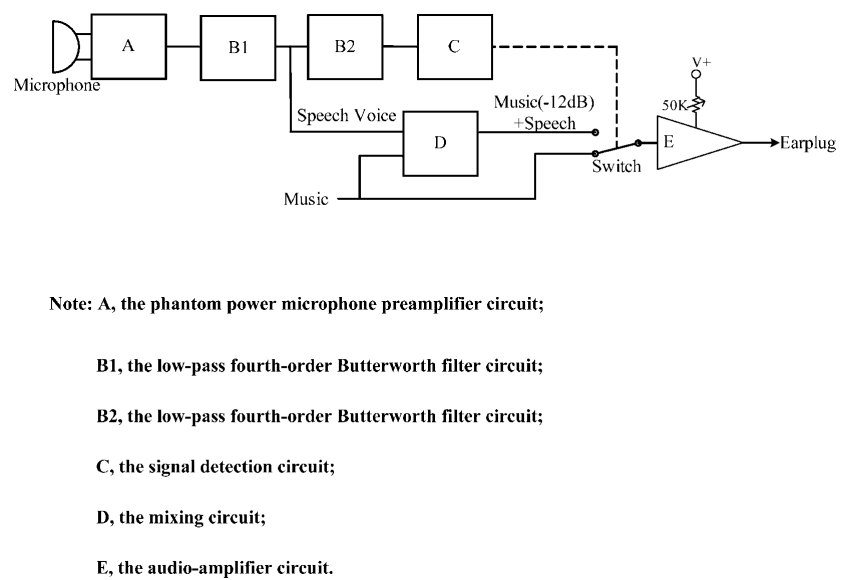

Fig. 1. A block diagram for the noise-filtering earplug system. and mental anxiety of patients with toothaches should be carefully evaluated since they usually present to dentists for further care. In fact, preventing exposure to noise can be appropriately combined with the controlled use of sound and music as further proposed by Chlan [9]. This approach would not only succeed in reducing a patient's exposure to noise, but would also utilize the therapeutic advantages of music. Due to the personal nature of one's noise threshold and music preferences, the use of a noise-filtering earplug system with available music would be essential to effectively combine these two objectives; this idea also formed the basic motivation of the present study. In fact, this novel earplug system can be used to reduce extreme noise levels throughout dental treatments as well as provide a kind of music therapy, which was originally proposed for rehabilitation. At that time, approximately 13 years ago, research in music therapy covered areas such as music's effects on disabled patients, comatose patients, and senior patients as well as those on artificial respiration and for healing professional musicians [6]. To date, music therapy has shown beneficial effects in protecting the environment of newborns [5, 24] and operating rooms [22], as well as on those patients undergoing regional anesthesia [13] and those with postoperative pain [16]. In addition, positive effects were also reported for pregnancy in various stressful contexts [21] and children with severe burn injuries [12]. All in all, those studies indicate that using music and sounds to reduce pain and anxiety in neonate, pediatric, surgical, and adult patients within different hospital settings shows "sound" results. However, a description of a noise-filtering earplug system combined with music applied to dental treatments for reducing noise levels, pain, and anxiety as well as attention deficits was not found with the authors' best efforts.

Based on the motivations described above, we first collected and analyzed the frequency ranges of dental noises and audible sounds from a domestic dental hospital. The combined use of a personal computer (PC) with available software packages of CoolEdit and LabVIEW was adopted for off-line analysis to determine the appropriate cutoff frequency. Then, a digital wave-filtered approach was adopted to filter out the dental noise and reserved the frequency range of speech. After the reducing effects were identified, a self-designed digital wave-filter was developed on a circuit board as an auxiliary noise-filtering tool. The developed earplug system, unlike a conventional ear-mask only used for preventing the noise and able to be adjusted according to the different treated teeth of a patient, is more convenient owing to the smaller size of the earplug. In addition, along with the function of reducing noise levels, a small microphone and an available 
MP3 music player are incorporated in the earplug system to help the patient better relax from anxiety and enhance communication between the dentist and patient during treatment. Fig. 1 is a block diagram of the developed noise-filtering earplug system. In this figure, when the signal is detected to be higher than a threshold value, the controlled electric circuits will automatically reduce the music signal by $6-10 \mathrm{~dB}$ or cut off the music signal for speech purposes.

The present paper, apart from the present section, is organized as follows. Section 2 describes experimental setups and theoretical backgrounds for the developed noise-filtering earplug system. In Section 3, we illustrate the functions and elements of the design system in detail. Results are checked and discussions are provided in Section 4. Finally, Section 5 concludes with some important remarks on reported results.

\section{EXPERIMENTAL SETUPS AND THEORETICAL BACKGROUNDS}

\section{Dental noise collection}

Since the wave filter is adopted for noise reduction, the advance collection of dental noise signals was quite important so that the frequency ranges of the noise and speech signals could be classified. Considering the inconvenience of a direct signal analysis at the collection location, those signal data were recorded with a type AT9360 microphone on a mini-disk recorder from Sony. The mini disc, instead of a tape, was used to store digitized signals from the microphone with a sampling rate of $44.1 \mathrm{kHz}$, and it has the same quality as a compact disc (CD). Thereafter, an SPDIF (Sony/Philips Digital Interface) connected with the disc recorder transmitted the recorded digital signals to the $\mathrm{PC}$ in the laboratory for further analysis. Since data acquisition can reach 24 bits $/ 96 \mathrm{kHz}$, signal aliases can be reduced to a minimum. It should be noted that the quality of the signal acquisition by the microphone depends on its directivity. This means that the direction of travel of the sound waves, whether perpendicular or parallel to the diaphragm, striking the diaphragm of the microphone influences the microphone's response. If the sound to be measured arrives at the microphone from a predominant direction, then one of these two preferred directions should be used. In general, perpendicular-incidence is recommended for stationary sound sources while parallel-incidence, also called [8] grazingincidence, is used for moving sound sources so that the same sound incidence angle is present at all times throughout the measurement period. In this case, a perpendicular-incidence response was adopted. With a narrow directivity pattern for the AT9360 and available frequency response range of 100 to $15,000 \mathrm{~Hz}$, which covers nearly the entire audible range of the human ear, data acquisition with this setup can avoid background noise.

\section{Application of power spectral density}

To identify the frequency ranges of the noise from dental machines as well as those of speech, the collected digital signals in time domain need to be transformed to frequency domain by using the fast Fourier transformation (FFT) method. Throughout the present study of digital signal processing, the frequency domain played an important role in the design and analysis of signals and the system. To investigate the effects of wave filtering, the power spectral density in the frequency domain was adopted to calculate the finite energy of signals spread over the spectrum. Now, assuming that $x_{k}$ represents a set of real signal sequences, $X_{m}$ denotes the Fourier transformation of $x_{k}$, and $N$ (an even number) is the sampling number, then, the power spectra density, $P_{m}$, can be expressed as [8]

$$
P_{m}=\frac{1}{N}\left|X_{m}\right|^{2}, m=0,1,2, \ldots, N-1,
$$

where

$$
X_{m}=\sum_{k=0}^{N-1} x_{k} e^{-j(2 \pi k m / N)} .
$$

The total energy of signals can be yielded as

$$
E_{t}=\frac{1}{N^{2}} \sum_{m=0}^{N-1}\left|X_{m}\right|^{2}=\frac{1}{N} \sum_{m=0}^{N-1} P_{m} .
$$

\section{Determination of the cutoff frequency}

The noise signal was collected from local dental hospitals, and machine noises included those produced from hand-pieces of high and low speeds, high-power suction, scaling equipment, and speech. When recording, the microphone was about $20 \mathrm{~cm}$ from the noise sources for noise level calculation. A sample of the waveforms for the noise signal of a low-speed hand-piece and speech in time and frequency domains are shown in Figs 2(a) and 2(b), respectively. It should be noted that Fig. 2(b) shows a noise frequency range of 0 to $20,000 \mathrm{~Hz}$ and Fig. 3 shows most of the frequency range of the speech signal is within $1,000 \mathrm{~Hz}$ after the filter has been adopted. Under such a condition, we found that both the patient and dentist could communicate each other during the clinic experiment; therefore, the cutoff frequency was initially determined to be $1,000 \mathrm{~Hz}$. A $1,000-\mathrm{Hz}$ low-pass filter, which means the low-frequency components are retained and the high-frequency components are discarded, was adopted to filter out the 
noise. The retained frequency components were also transformed into a file of a wave format for instantaneous illustration of the filtering effects shown on a PC.

\section{Testing of noise filtering}

Determining how to filter the noise effectively and make the mechtronic mechanism into the designed circuit was part of the main concerns for this study. A second-order low-pass Butterworth filter is usually selected since it is called a maximally-flat-magnituderesponse filter and is optimized for gain flatness in the pass-band. Furthermore, the transient response of a Butterworth filter to a pulse input shows moderate overshoot and ringing. In addition, a fourth-order lowpass filter can be composed of two second-order lowpass filters in practice. As for choosing the secondorder or fourth-order low-pass filter, it depends on the signal filtering effect. More specifically, it related with the roll of rate of frequency response of signals with respect to the choice of the second-, fourth- or eighthorder filter. To design the filter circuit, the transfer function was needed to build the circuit and related theoretical backgrounds can be found in Ref. [20].

To further determine whether $1,000 \mathrm{~Hz}$ is a suit-

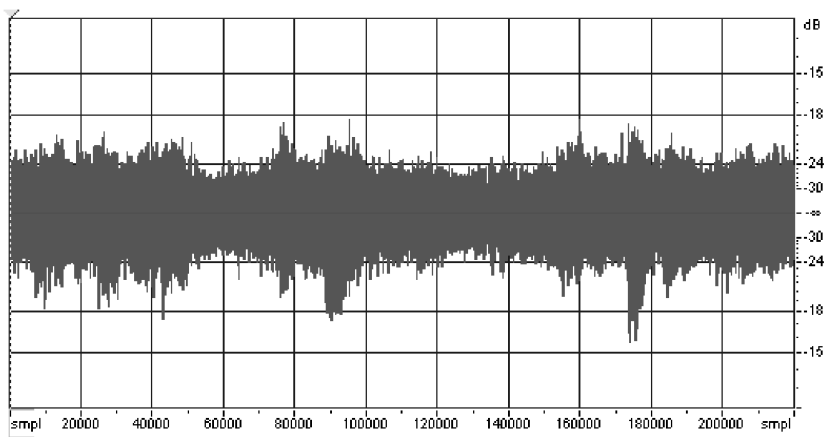

(a)

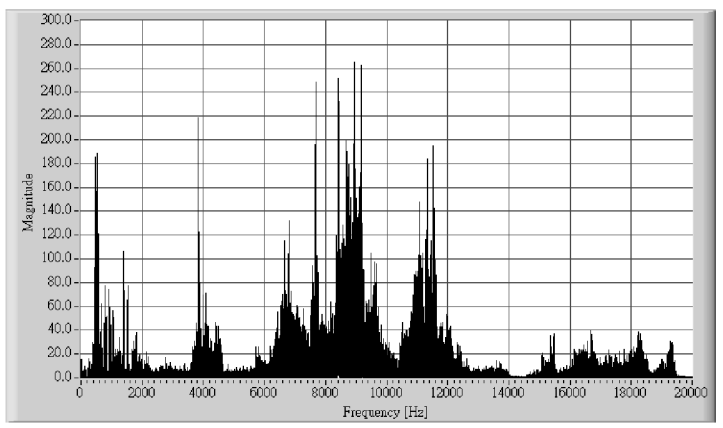

(b)

Fig. 2. Noise signals of a low-speed hand-piece and speech in time and frequency domains. able cutoff frequency for this study, a series of cases using $600,800,1,200$, and $1,400 \mathrm{~Hz}$ as the cutoff frequency with the same collected signals and filter was selected for testing. The results are shown in Fig. 4(a)4(d). Comparisons of the four figures found that most frequencies of speech signals are within $600 \mathrm{~Hz}$. If the cutoff frequency is set too high, then less of the noise signal will be filtered out, and the effects of filtering will be reduced. In contrast, if the cut-off frequency is set too low, too much of the speech signal might be filtered out, and speech will not dominate. Therefore, setting $1,000 \mathrm{~Hz}$ as the cutoff frequency was determined to be appropriate in this case of clinic experiment. In addition, when the amplitude of those signals was converted into sound pressure level (in $\mathrm{dB}$ ), the effect of the filter at the cutoff frequency of $1,000 \mathrm{~Hz}$ greatly dominated, and the results are shown in Figs. 5(a)-5(b). Moreover, a switch of available cutoff frequencies is also designed and attached with this earplug system for the users in case of adjustment of communication quality.

\section{FUNCTIONS AND ELEMENTS OF THE DESIGN SYSTEM}

The block diagram of the developed earplug system for filtering out dental noises is shown in Fig. 1 and we briefly introduce the kernel elements of the system with their respective functions below.

\section{Preamplifier circuit for the microphone}

A Phantom Power circuit design was adopted for the preamplifier circuit to increase the speech signal collection effect. The design combines the two connectors of the capacitor-type microphone with the external circuit and thus, they become a three-terminal microphone, as shown in Fig. 6. The advantage of such a design is that changing the signal transmission of the microphone to a differential type leads to an in-

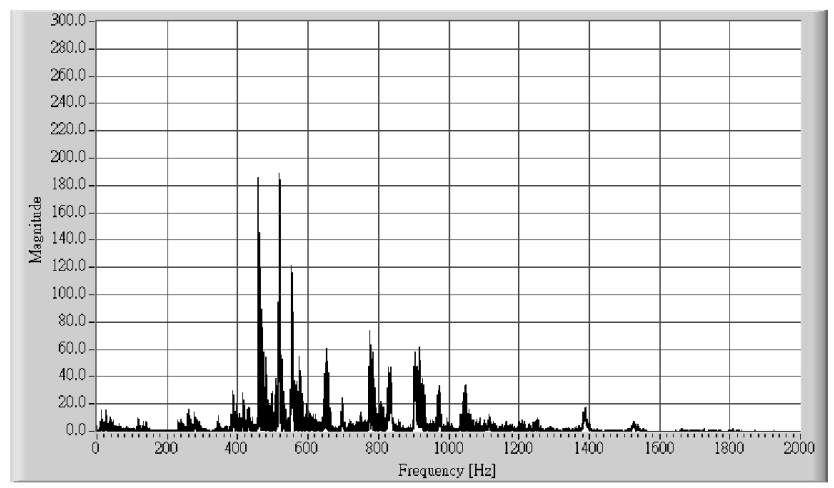

Fig. 3. A sample of the speech signal after using the filter. 
creased signal-to-noise ratio. More specifically, adding a fixed direct voltage at the two differential points of the microphone supplies the bias voltage of the microphone. Thus, when the noise signal due to air disturbances is received, it makes the capacitive reactance of the capacitor-type microphone change, and two signals will exist with equal amplitudes but opposite phases at the inputs of the amplifier. Due to the opposite phases of the two differential signals, the

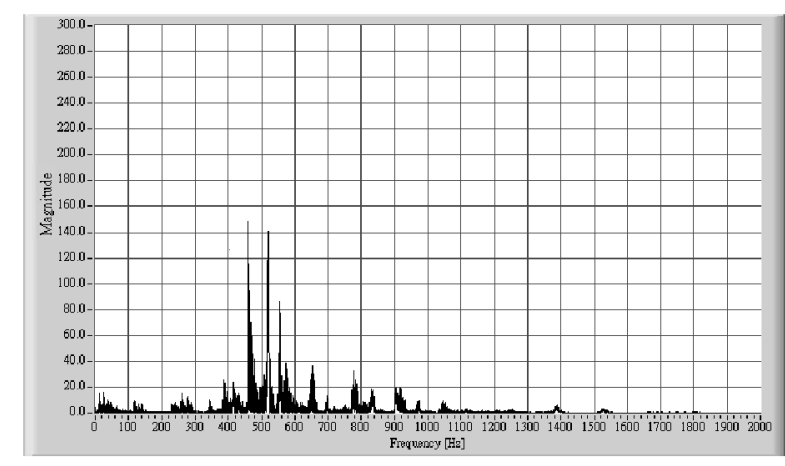

(a) Cutoff frequency at $600 \mathrm{~Hz}$

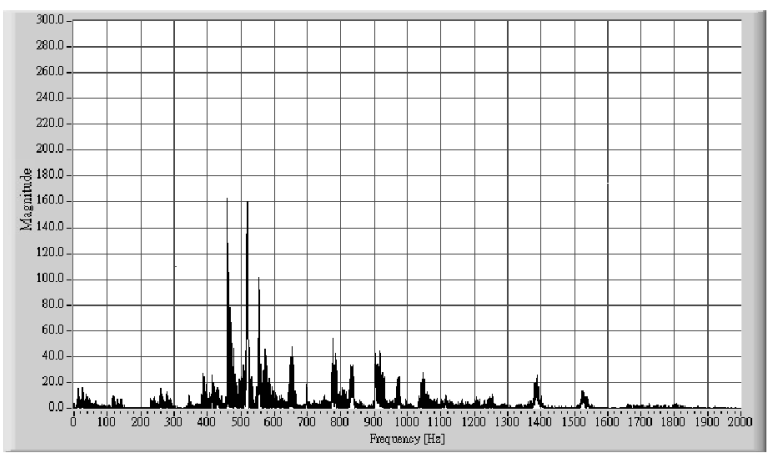

(c) Cutoff frequency at $1,200 \mathrm{~Hz}$ amplitudes of the signals will be doubled, and the "signal noise" with the same phase at the inputs of the amplifier will be canceled out. The amounts of the diminished signal noise depend on the composition of the two resistors, R6 and VR1, as well as the capacitor, C5, as shown in Fig. 6. Moreover, the rectangular block on the right of this figure, U1, is a set of three OP-AMPs with the IC number of INA 128 to form the instrument amplifier.

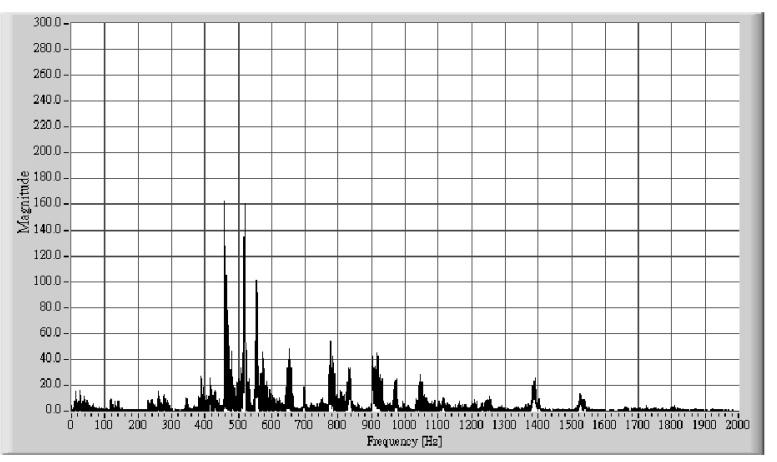

(b) Cutoff frequency at $800 \mathrm{~Hz}$

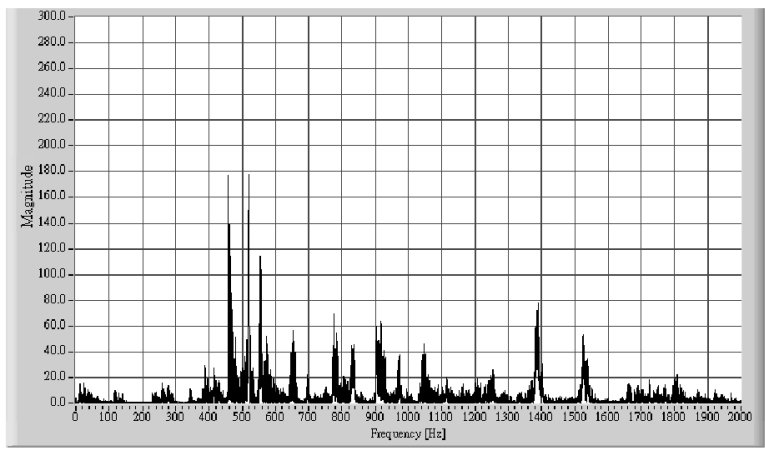

(d) Cut-ff frequency at $1,400 \mathrm{~Hz}$

Fig. 4. Signal results after using the filter for cutoff frequencies at $600,800,1,200$ and $1,400 \mathrm{~Hz}$.

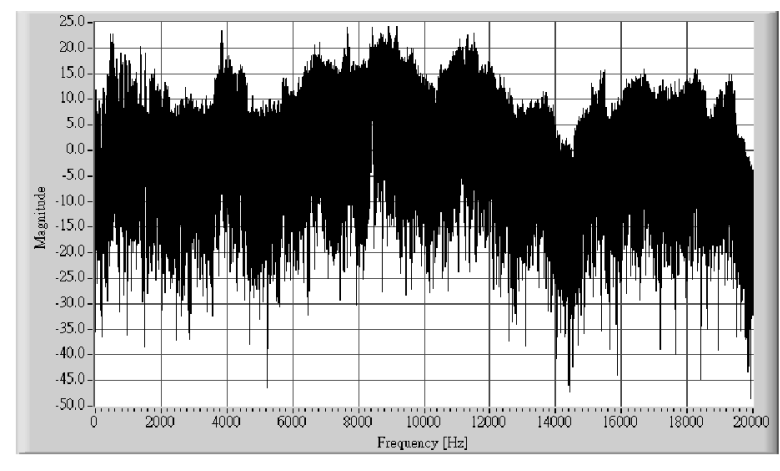

(a) Noise distribution before filtering

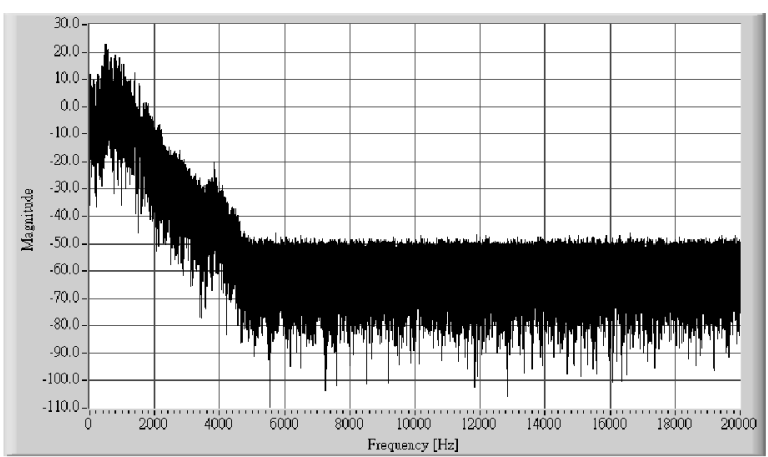

(b) Noise distribution after filtering

Fig. 5. Effect of the filter with a cutoff frequency at $1,000 \mathrm{~Hz}$. 


\section{Low-pass filter}

As shown in Fig. 7, a low-pass fourth-order Butterworth filter was adopted for the filter circuit due to its maximally-flat-magnitude-response; it is also called a maximally flat filter. A fourth-order filter can produce attenuation of $-80 \mathrm{~dB} /$ decade $(-20 \mathrm{~dB} / \mathrm{dec}$ ade per pole), and the needed parameters for operation can be calculated and obtained using the available package, FILTER-PRO, which is a low-pass filter design program issued by Texas Instruments (TI). The program also offers two types of circuits and three kinds of filters for selection. With appropriate values of capacitors, the calculated values of components of the circuit with the designed filter circuit can be simultaneously displayed. The fourth-order low-pass filter for the circuit in this case is also adopted to fulfill the requirements of the package.

\section{Signal level detection circuit}

Due to accuracy concerns, when designing the signal level detection circuit shown in Fig. 8, the signal mean value of the integrated circuit must be considered so that it can avoid the false operation of the circuit especially when a single impulse of signal noise is encountered. In addition, a Schmitt trigger circuit was also adopted in the level criteria circuit to determine the critical range as an operational area since it can avoid false operation due to the continuous switching of outputs of the detection circuit when speech has a large variation.

\section{Mixing circuit and audio-amplifier circuit}

A mixing circuit shown in Fig. 9 was designed to partially reduce the music signal when communication between the dentist and patient is necessary. However, at that time, the output of the earplug is purely the received signal of the microphone, and there may be a large difference in the receiver and the earphone. Therefore, a reduction of $12 \mathrm{~dB}$ in the music signal was considered and put into practice; this makes the patient with the earplug system feel more comfortable even if some interference exists when the system is operating.

The audio-amplifier circuit shown in Fig. 10 was

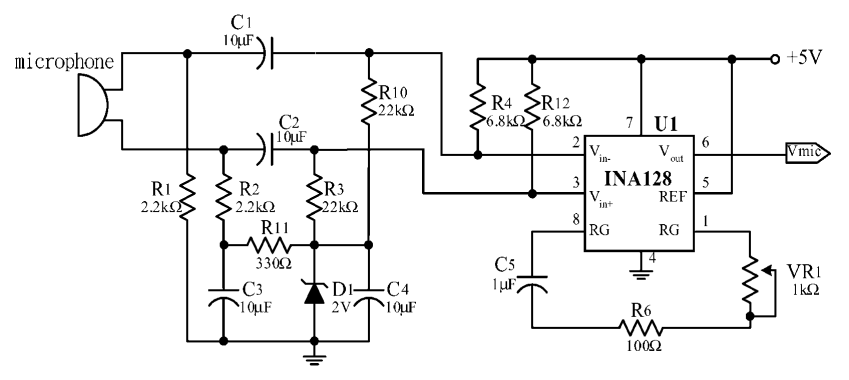

Fig. 6. Phantom power microphone pre-amplifier circuit.

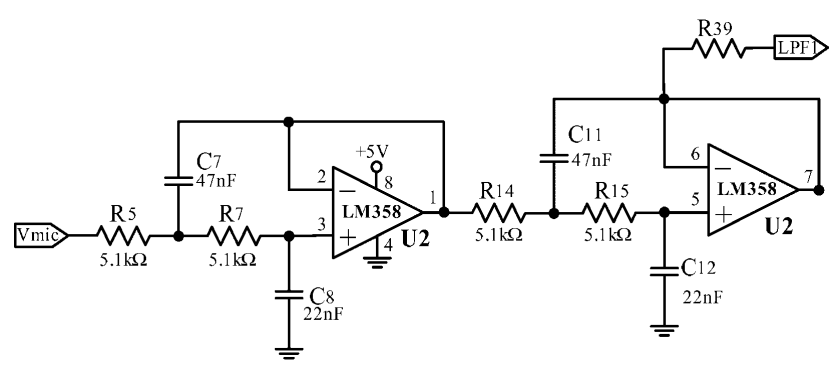

Fig. 7. Low-pass fourth-order Butterworth filter.

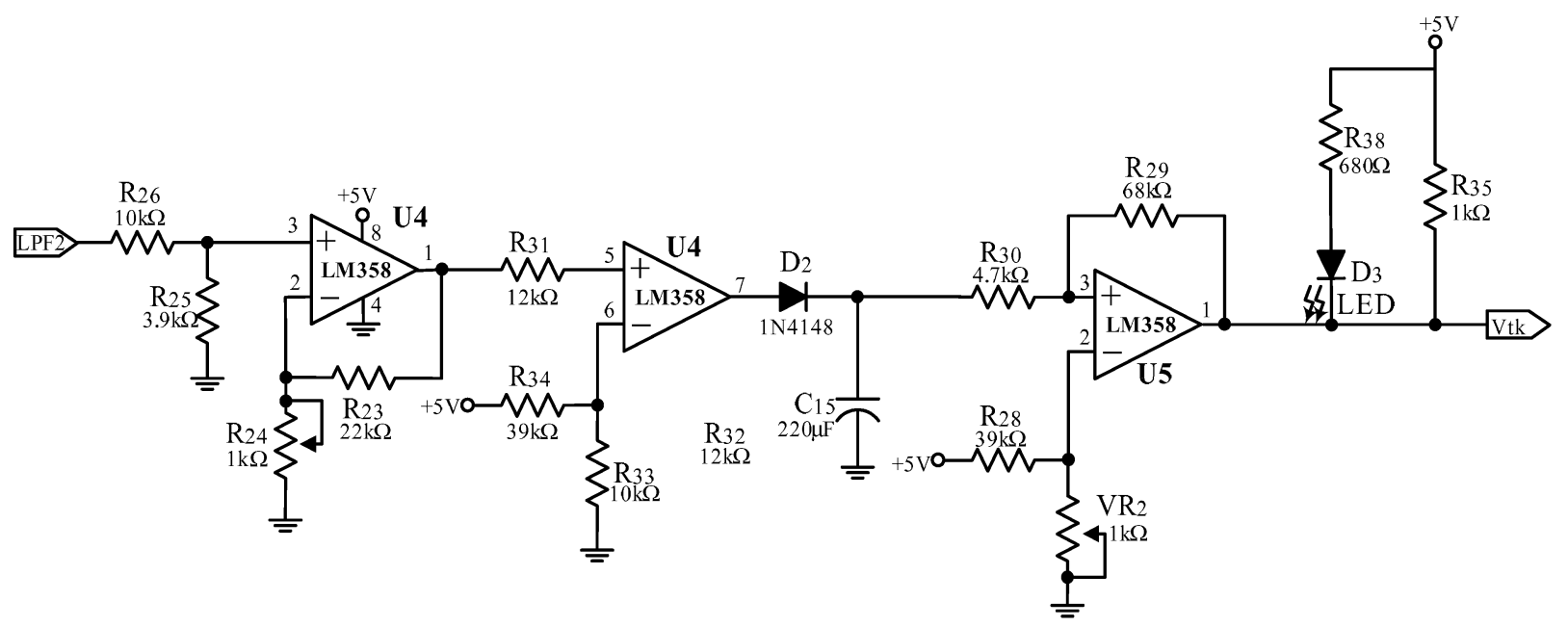

Fig. 8. Signal detection circuit. 
adopted directly from an available audio-amplifier (TPA0232) in an integrated circuit (IC) from TI for convenience. With advantages of multi-functional control and a switch for two different sound sources, it meets the requirements of the current system. Apart from these advantages, no sound impulse exists when the electrical switch is operating, and output power of 2 $\mathrm{W}$ is sufficient to operate the earphone.

\section{Earplug with an earphone and PCB fabrication}

The MDR-NC11 product from Sony was adopted due to its small size and because the earplug has an available built-in microphone. When the digital filter circuit is integrated into the system, it meets the requirements of the designed system. After testing all the circuits, a package with the Windows version of PCAD

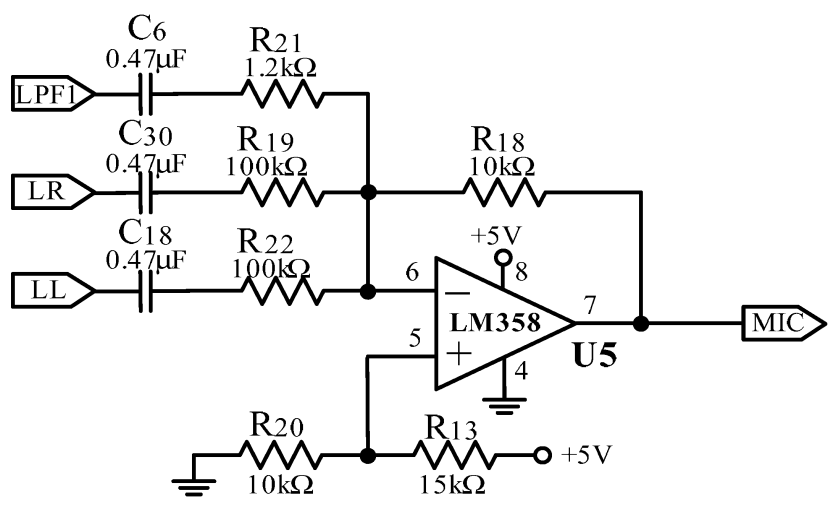

Fig. 9. Mixing circuit. was used to make the circuit arrangements on the printed circuit board (PCB). For reducing the size and commercial practice of the $\mathrm{PCB}$, large amounts of surface mate device (SMD) components were adopted for the circuits. The general arrangement of the circuits can be converted into a Gerber file format and then, directly transmitted to the carving machine for fabrication.

\section{TESTING RESULTS AND DISCUSSIONS}

\section{Adjusting the circuits}

For reducing the size of the circuit as much as possible and increasing its stability, the IC made from TL128 was replaced by an INA128 in the microphone preamplifier circuit. In addition, when testing, a variable resistor was also included in the circuit to provide the maximum electric output without a signal alias. An additional fourth-order filter was also introduced in the signal level detection circuit to reduce the occurrence of false operation due to a sudden signal after testing. Moreover, the microphone signal from the fourth-order filter was also amplified in the mixing circuit to control the sensitivity of the signal level detection circuit by adjusting the amplifier.

\section{Testing the circuits}

Two kinds of equipments, a frequency response recorder (LEADER, LFR-5601) and a Tektronic oscilloscope (TDS3034) with an FFT tool, were used to test the circuits. The former was used to illustrate the

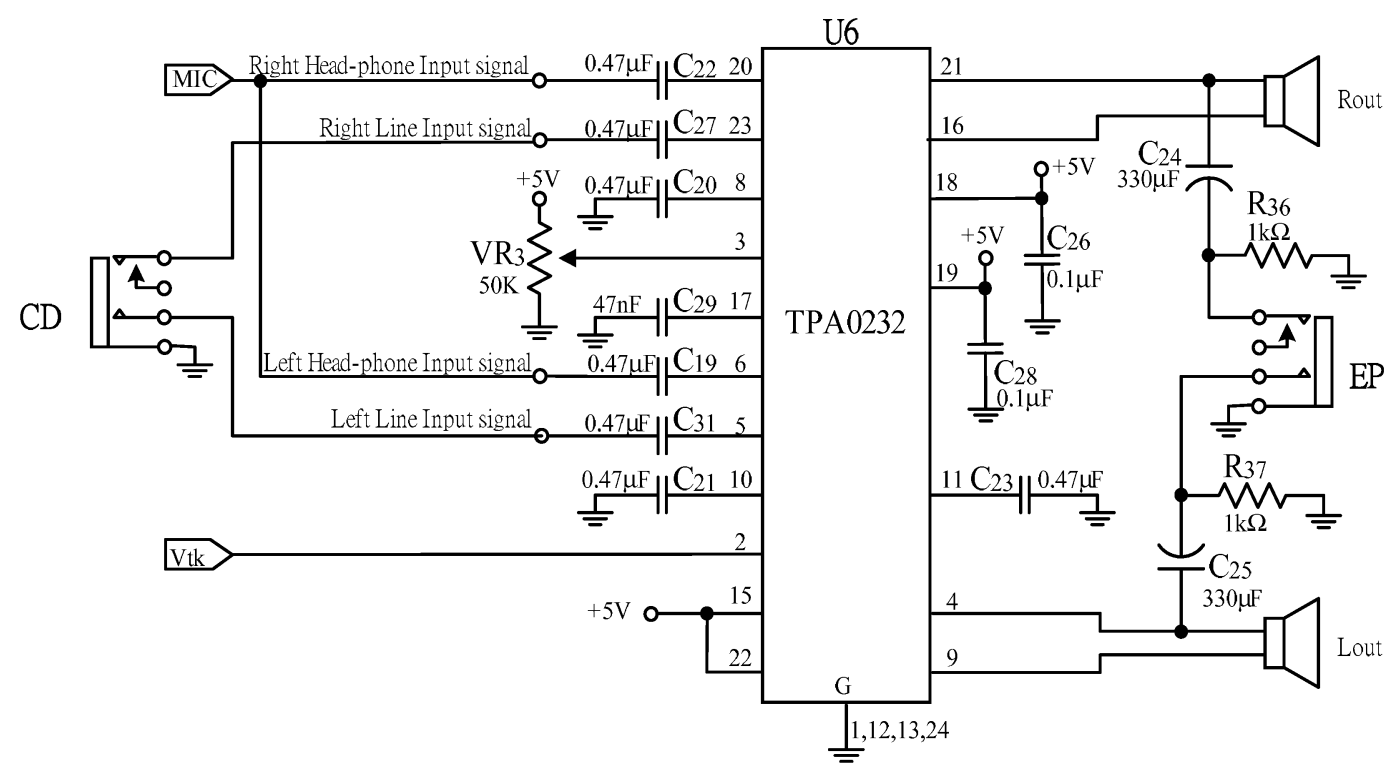

Fig. 10. Audio-amplifier circuit. 


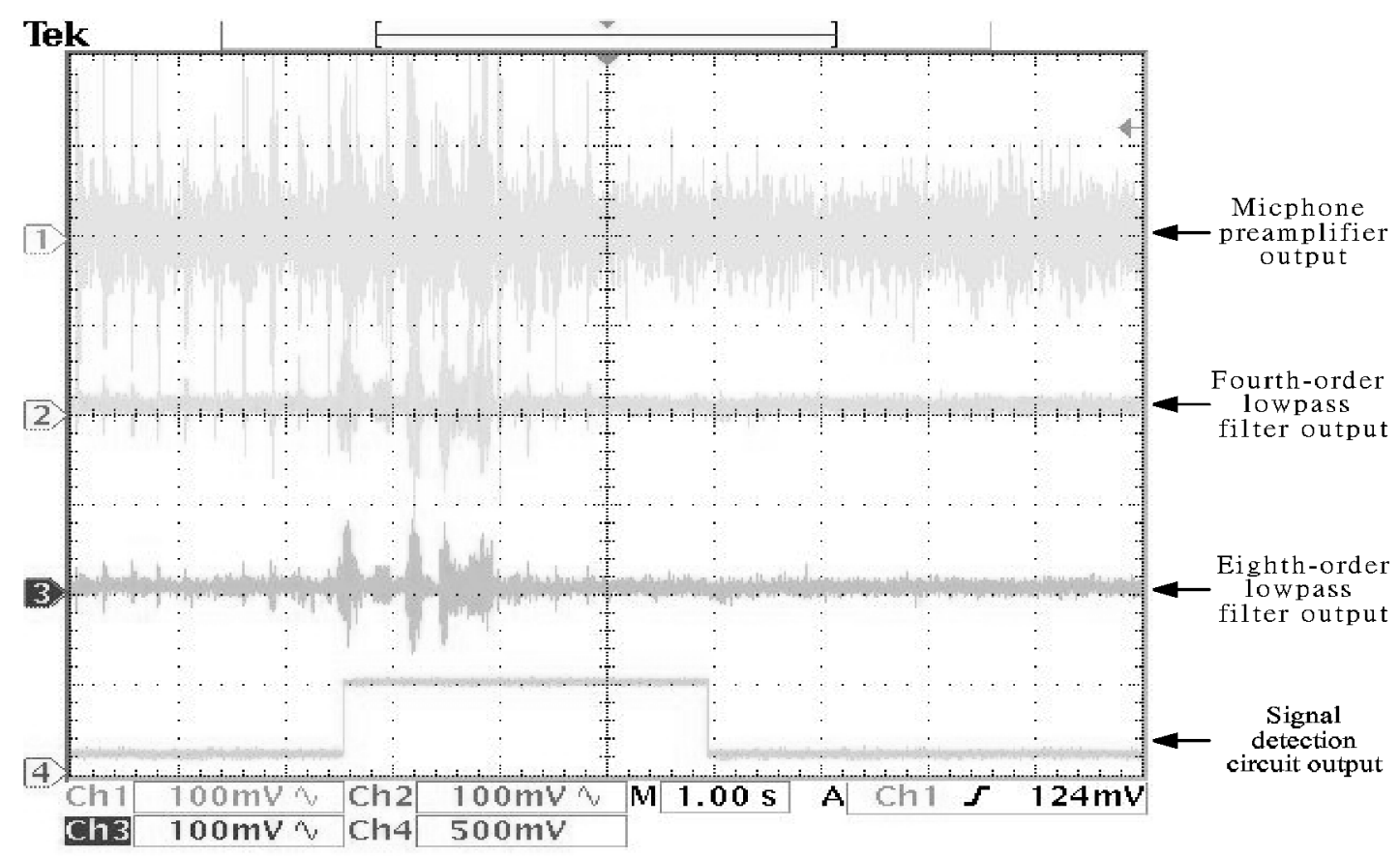

Fig. 11. Various output signals through different circuits from the dental signals.

frequency response curves of all used circuits, while the latter was adopted to test the frequency response of all circuits. The external signals were transmitted by the speaker (AWAI SC-B10H) to the microphone at a distance of $20 \mathrm{~cm}$. The external signals were categorized into a standard signal such that the pink noise ranged from 100 to $10,000 \mathrm{~Hz}$ and the dental noise signal were recorded in an off-line format for simulation. After testing, characteristic curves of circuits for the microphone preamplifier and the fourth-order low-pass filter both show a good condition. As for the frequency response functions obtained by using the FFT tool, when the standard and dental noise signals have input, test results show that after adjusting the circuit, the operating conditions for each element of the developed earplug system were good as shown in Fig. 11.

\section{Music from MP3}

It is well known that music can play an important role in relaxing the anxiety and tension of patients. Some dental hospitals also offer music to patients. However, a public broadcast system is usually used to present the music, and it has disadvantages of being interfered with by unwanted sounds, and the patient has no say in the selection. Therefore, in our design, the music is broadcast through an earplug system, and an evaluation test was conduced by using a Hemo Dynamic Monitor from Hewlett-Packard with sensors attached to the patient to measure blood pressure and heart rate. However, the effects from the two indexes were not dominant, although most patients claimed that the noise had been reduced.

\section{Discussion}

Some proposals from circuit testing are given as follows. The 8-pin INA128 amplifiers, instead of the 16-pin TL084 type, which were adopted in the audioamplifier circuit offered excellent accuracy due to their low power and general- purpose instrumentation. Their versatile 3-op amp design and small size make them ideal for a wide range of applications. Current-feedback input circuitry provides wide bandwidth even at high gain $(2,000 \mathrm{~Hz}$ at $\mathrm{G}=100)$. Meanwhile, a single external resistor set the gain from 1 to 10,000, and the INA128 provides an industry standard gain equation. Moreover, the INA128 is laser-trimmed for a very low offset voltage of $50 \mu \mathrm{V}$, draft at $0.5 \mu \mathrm{V} /{ }^{\circ} \mathrm{C}$, and high common mode rejection of $120 \mathrm{~dB}$ at $\mathrm{G} \geq 100$. It operates with power supplies as low as $\pm 2.25 \mathrm{~V}$, and a quiescent current of only $700 \mu \mathrm{A}$ is ideal for batteryoperated systems. Finally, internal input protection can withstand up to $\pm 40 \mathrm{~V}$ without damage and these merits make the earplug system easy to place into commercial practice.

The signal output from the fourth-order filter was originally directly transmitted to the signal detection 
circuit and the mixing circuit; however, an impulse signal, such as a cough, may result in false operation. Therefore, after testing, a fourth-order filter was included in the system before the signal was transmitted to the detection circuit to reduce possible occurrences of false operation. This makes the function of the system more practical for various applications. In addition, another variable resistor $\left(\mathrm{VR}_{2}\right)$ at $\mathrm{U} 5$ of the detection circuit, by which the operation of the entire system is initiated, was also included to adjust the trigger level; this makes the function of the detection circuit more flexible. Moreover, when the speech signal is triggered in the system, the music signal is automatically reduced by $12 \mathrm{~dB}$. After passing through the fourth-order filter for filtering out unnecessary noise, the speech signal can also be amplified again in the mixing circuit, facilitating communication between the patient and dentist.

Finally, we found that most dental hospitals have an open area for treatment. If the sensitivity of the signal trigger value is set too high, then communication interference may result. In addition, when the speech of the dentist is too soft, the communication effect will be reduced due to the earplugs on the patient. An earplug with a wireless microphone for the dentist is suggested to increase communication quality, and a circuit is now being designed for this. Meanwhile, considering the effects of the music, the music broadcast system can be set up in the dental chair to reduce the interference between the music source and the receiver. Studies on optional music selection combined with music therapy should be conducted.

\section{CONCLUSIONS}

In this paper, a newly developed earplug system for filtering out dental noise is reported which resolves noise problems in the working environment of the dentist. In addition, with music in an MP3 format included with the earplug system, it not only can serve as a communication tool between the dentist and the patient but also offer an effective solution to relieve the anxiety and tension of the patient during treatment. Testing results show that the developed earplug system can be fabricated for commercial applications due to its size and economy. If music therapy can be further included in the musical element, further medical applications are possible. A cross-sectional study using a self-reporting questionnaire for local dental hospitals has been undertaken. The results of that study will be reported soon.

\section{REFERENCES}

1. Arvidsson, O. and Lindvall, T., "Subjective Annoyance from Noise Compared with Some Directly Measurable Effects," Int. Arch. Occup. Environ. Health, Vol. 33, pp. 159-166 (1978).

2. Belojevic, G., Jakovljevic, B., and Aleksic, O., "Subjective Reactions to Traffic Noise with Regard to Some Personality Traits," Environ. Int., Vol. 23, pp. 221-226 (1997).

3. Belojevic, G., Slepcevic V., and Jakvoljevic, B., "Mental Performance in Noise: The Role of Introversion," $J$. Environ. Psychol., Vol. 21, pp. 209-213 (2001).

4. Berglund, B. and Lindvall, T. (eds.), "Community noise" Archives of the Center for Sensory Research. Stockholm University and Karolinska Institute, Stockholm, Vol. 2, pp. 58-67; pp. 79-82 (1995).

5. Burke, M., Walsh, J., Oehler J., and Gingras, J., "Music Therapy Following Suctioning: Four Cases Studies," Neonatal Netweek, Vol. 14, pp. 41-49 (1995).

6. Cabrera, I.N. and Lee, M.H.M., "Reducing Noise Pollution in the Hospital Setting by Establishing a Department of Sound: A Study of Recent Research on the Effects of Noise and Music in Health Care," Prevent. Med., Vol. 30, pp. 339-345 (2000).

7. Carter, N.L., "Transportation Noise, Sleep and Possible After-effects," Environ. Int., Vol. 22, pp. 105-116 (1996).

8. Cavicchi, T.J., Digital Signal Processing, John Wiley and Sons, Inc., Singapore, Ch. 10, pp. 673-777 (2000).

9. Chlan, L., "Effectiveness of a Music Therapy Intervention on Relaxation and Anxiety for Patients Receiving Ventilatory Assistance," Heart Lung, Vol. 27, pp. 169176, 1998.

10. Chowanadisai, S., Kukiattrakoon, B., Yapong, B., and Kedjarune, U., "Occupational Health Problems of Dentists in Southern Thailand," Int. Dental J., Vol. 50, pp. 36-40 (2000).

11. Clark, W.W. and Bohne, B.A., "Effects of Noise on Hearing," JAMA, Vol. 281, pp. 1658-1659 (1999).

12. Edward, J., "Music Therapy for Children with Severe Burn Injury," Music Therapy Perspect., Vol. 16, pp. 2126 (1998).

13. Eisenman, A. and Cohen, B., "Music Therapy for Patients Undergoing Regional Anesthesia," AORN J., Vol. 62, pp. 947-950 (1995).

14. Fahy, F., Foundation of Engineering Acoustics, Elsevier Science, Singapore, pp. 1-22 (2001).

15. Feinmann, C. and Harrison, S., "Liaison Psychiatry and Psychology in Dentistry," J. Psychosomatic Rev., Vol. 43, pp. 467-476 (1997).

16. Good, M., "Effects of Relaxation and Music on Postoperative Pain: A Review," J. Adv. Nursing, Vol. 24, pp. 905-914 (1996).

17. Gulian, E., "Psychological Consequences of Exposure to Noise, Facts and Explanations," In W. D. Ward (Ed.), Proceedings of International Congress on Noise as a Public Health Problem, U.S. Environmental Protection 
Agency, Dubrovnik, Washington, D.C., pp. 363-378 (1973).

18. Hygge, S., Jones D.M., and Smith, A.P., "Recent Developments in Noise and Performance," In N. Carter and R. F. S. Job (Eds.), Proceedings of the $7^{\text {th }}$ International Congress on Noise as a Public Health Problem, Sydney, Australia, pp. 321-328 (1998).

19. Jacobsen, N., Derand T., and Hensten-Pettersen, A., "Profile of Work-related Health Complaints among Swedish Dental Laboratory Technicians," Comm. Dentistry Oral Epidemiol., Vol. 24, pp. 138-144 (1996).

20. Karki, J., "Active Low-Pass Filter Design,” Application Report-SLOA049B, Texas Instruments, USA, pp. 1-21 (2002).

21. Liebman, S.S. and Maclaren, A., "Effects of Music and Relaxation on Anxiety in Adolescent Pregnancy," Proceedings of Current Research in Arts medicine, MedArt Int., Chicago, pp. 427-431 (1995).

22. Long, C., "Doctors Find Music Works Well with Sedatives and Anesthetics," Insight News, Vol. 12, pp. 41-42
(1996).

23. McLean, E.K. and Tarnopolsky, A., "Noise, Discomfort and Mental Health," Psychol. Med., Vol. 7, pp. 19-62 (1977).

24. Munson, M., "Plugged in Preemies: Tunes May Help Babies Breathe Better," Prevent., Vol. 47, pp. 42-43 (1995).

25. Prince, M.M., "Distribution of Risk Factor for Hearing Loss: Implications for Evaluating Risk of Occupational Noise-induced Hearing Loss," J. Acoust. Soc. America, Vol. 112, pp. 557-567 (2002).

26. Smith A.P. and Miles, C., "The Combined Effects of Occupational Health Hazards: An Experimental Investigation of the Effects of Noise, Nightwork and Meals," Int. Archi. Occup. Environ. Health, Vol. 59, pp. 83-89 (1987).

27. Wu, T.N., Hwang, J.T., Chu P.E., and Chang, P.Y., "Effects of Noise Exposure and Task Demand of Cardiovascular Function," Int. Archi. Occupa. Environ. Health, Vol. 60, pp. 99-105 (1988). 